\title{
Surface Plasmon Polaritons and Their Role in the Enhanced Transmission of Light through Periodic Arrays of Subwavelength Holes in a Metal Film
}

\author{
W. L. Barnes, ${ }^{1}$ W. A. Murray, ${ }^{1}$ J. Dintinger, ${ }^{2}$ E. Devaux,${ }^{2}$ and T.W. Ebbesen ${ }^{2}$ \\ ${ }^{1}$ School of Physics, University of Exeter, Stocker Road, Exeter, United Kingdom \\ ${ }^{2}$ ISIS, Université Louis Pasteur, 8 rue Gaspard Monge, Strasbourg 67000, France
}

(Received 17 June 2003; published 9 March 2004)

\begin{abstract}
We present results of the transmitted, reflected, and absorbed power associated with the enhanced transmittance of light through a silver film pierced by a periodic array of subwavelength holes. Comparing experimentally acquired dispersion curves under different polarization conditions shows that the transmission features of the array are consistent with $p$-polarized resonant modes of the structure. By exploring the regime in which no propagating diffracted orders are allowed, we further show that the transmittance maxima are associated with both reflectance minima and absorption maxima. These new results provide strong experimental evidence for transmission based on diffraction, assisted by the enhanced fields associated with surface plasmon polaritons.
\end{abstract}

PACS numbers: 78.20.Ci, 41.20.Jb, 42.25.Fx, 73.20.Mf

The observation of the enhanced transmission of light through a metallic film perforated by an array of subwavelength holes [1] sparked a wealth of research seeking to explain the underlying physics. For light of wavelength $\lambda$, a hole less than $\sim \lambda / 2$ in diameter in a thick metal film does not support any propagating modes; energy can propagate only by an evanescent tunneling process leading to very weak transmittance - in marked contrast to the experimental observations.

Initial explanations considered coupling of the incident light via diffraction to surface plasmon polariton (SPP) modes of the metallic structure [1,2]. Many different theoretical approaches have since been adopted, all replicating the normal incidence transmission spectra [3-7]; consequently, more than just the normal incidence transmission spectrum needs to be explored if one is to obtain a clearer picture of the underlying physics. Despite a convergence of views among many theorists that the enhanced transmission involves multiple diffraction enhanced by SPPs [5-9], there remains some controversy surrounding the transmission mechanism; not all authors agree that surface plasmons are involved. (Note that explanations based on results from 1D arrays of slits cannot be used, and the physics is very different; sub- $\lambda / 2$ slits can support propagating modes, while sub- $\lambda / 2$ holes cannot [10].) As noted elsewhere [8], the experimental evidence obtained thus far is consistent with the involvement of SPPs rather than conclusive about their involvement. We present new experimental data not just of the transmittance, but also of the reflectivity and absorbance. We investigate two unexplored far-field signatures of SPPs in this context, their polarized nature and their dissipative character; we are thus able to identify the role played by SPPs in the transmission process.

Diffraction is central to the transmission process. Diffraction of the incident plane wave by the array produces waves with the required evanescent character; dif- fraction of the transmitted evanescent wave on the far side of the array then produces the propagating transmitted light. However, these diffraction processes are independent of the material from which the array is fabricated, whereas it has already been shown that good metals show higher transmittance than poor ones [11]. Therefore, although diffraction is central to the transmission process, the transmission in metallic structures is being enhanced in some way.

In our view, the key to the enhancement comes when diffraction is such as to allow coupling between the incident light and SPP modes; this may occur in three ways. (i) Incident light couples to an SPP mode supported by the surface facing the incident light. The enhanced field associated with an SPP mode increases the probability of transmission through the holes, where it is again scattered by the periodic array to produce light. (ii) Incident light cannot couple to an SPP mode on the incident side, instead matching conditions allow light that is weakly transmitted through the array to couple to an SPP mode on the far side; the enhanced electric field associated with the SPP mode increases the probability of transmission, and subsequent scattering again results in transmitted light. (iii) Matching conditions allow processes (i) and (ii) to take place simultaneously.

When the frequency, $\omega$, and in-plane wave vector, $k_{\|}$, of light that has been scattered by one or more grating vectors matches that of a mode of the structure both the transmittance and reflectance are modified. The matching condition is

$$
\mathbf{k}_{0} \sin \theta \pm i \mathbf{G}_{x} \pm j \mathbf{G}_{y}=\mathbf{k}_{\text {mode }}
$$

where $\mathbf{k}_{0}$ is the wave vector of the light incident at a polar angle $\theta, \mathbf{G}_{x}$ and $\mathbf{G}_{y}$ are the Bragg vectors associated with the two periodicities of the array, and $i$ and $j$ are integers indicating the order of the scattering event that couples 
incident light and a mode of the structure with wave vector $\mathbf{k}_{\text {mode }}$. By obtaining optical data as a function of incident frequency and in-plane wave vector, the dispersion of any modes involved may be ascertained [12]. We acquired such data for reflectance and transmittance as described elsewhere [13]. Briefly, samples were illuminated by a collimated $\left(\Delta \theta \sim 0.5^{\circ}\right)$, spectrally filtered beam $(\Delta \lambda \sim 2 \mathrm{~nm})$. Measurements of the transmitted/ reflected intensity were then recorded as a function of the incident wavelength and angle.

Samples were formed in $180 \pm 5 \mathrm{~nm}$ silver films deposited on silica glass substrates by thermal evaporation. Focused ion-beam lithography (FEI DB235) was used to mill the arrays. A period of $420 \pm 5 \mathrm{~nm}$ and a hole diameter of $250 \pm 5 \mathrm{~nm}$ ensured the holes were small enough to be sub- $\lambda / 2$ for the lowest energy transmittance peak $(\sim 710 \mathrm{~nm})$, but large enough to exhibit high transmittance and thus good contrast in the reflectivity data. Samples of area $1 \mathrm{~mm}^{2}$ were produced by tessellating a large number of smaller arrays, account being taken of the resulting diffraction.

Transmittance data as a function of frequency and inplane wave vector, for unpolarized incident light, are shown in Fig. 1(b), together with a normal incidence spectrum [Fig. 1(a)]. These data are similar to those obtained by Ghaemi et al. [12] and show the dispersion of the transmittance features. To within experimental error, we found the transmittance of light incident from

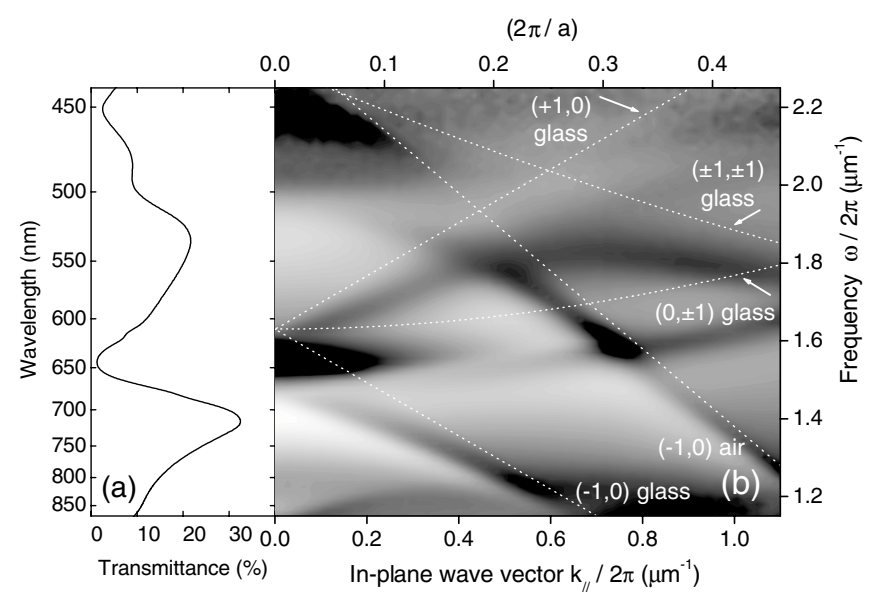

FIG. 1. Measured transmittance of the silver hole array for unpolarize light. To the left (a) is the normal incidence transmittance spectrum. To the right (b) transmittance is displayed as a function of in-plane wave vector and frequency in the form of a grey-scale dispersion diagram; black is low transmittance, white is high, and the data in (b) are shown on a logarithmic scale [the array period is (a)]. Also shown are the light lines (dotted lines) where diffracted orders become tangent to the surface; the different diffracted orders are indicated according to whether they occur in the substrate (glass) or air. [These light lines can be found by replacing $\mathbf{k}_{\text {mode }}$ in Eq. (1) with the value 0.] the air and substrate (glass) side of the sample to be the same, as expected from the reciprocity theorem [14].

A key feature of SPPs is that the electric field associated with them is polarized $[15,16]$. Transmittance dispersion data were acquired for $p$ - and $s$-polarized light (Fig. 2) and indicate a marked polarization dependence consistent with the involvement of SPPs; but what of their dispersion? To explore this we indicate as solid lines the calculated dispersion of the SPP modes of planar silver/ air and silver/glass interfaces. To calculate the modal dispersion, we used a well-established technique that incorporated the complex refractive indices of the materials involved together with their dispersion [17,18]. For both the $p$ - and $s$-polarized data the theoretically derived mode is offset to higher frequency than the experimental data.

Two observations can be made concerning the $s$-polarized transmittance data. First, the transmittance at normal incidence is independent of polarization, as expected from the symmetry of the array. Second, the $s$-polarized transmittance peaks exhibit very limited dispersion, matching that expected for SPP modes that can
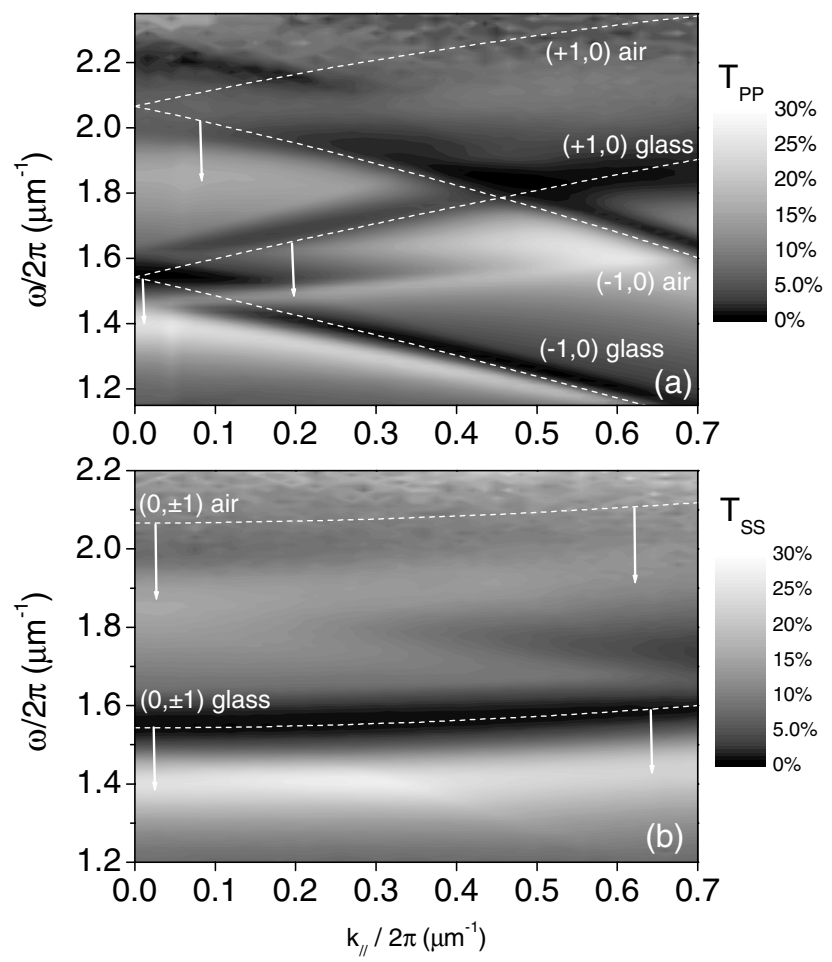

FIG. 2. Measured transmittance as a function of in-plane wave vector and frequency in the form of a grey-scale dispersion diagram for $p$-polarized incident light (a) and $s$-polarized incident light (b). Also shown are the calculated SPP dispersion curves for planar surfaces. Data are shown on a linear scale and dark regions are the primary indication of coupling to modes. The arrows indicate the offset between the experimentally derived and the theoretically predicted mode dispersions. 
be coupled with $s$-polarized light, the $(0, \pm 1)$ silver/glass and $(0, \pm 1)$ silver/air SPP modes.

Most of the essential features of the $p$-polarized data, Fig. 2(a), can also be associated with incident light coupling to SPP modes. Again, dashed lines indicate the calculated dispersion of the SPP modes. Coupling of incident light via $( \pm 1,0)$ scattering to the silver/glass SPP mode is quite clear, though offset slightly from the predicted feature. The dispersion of the transmittance feature associated with the silver/air SPP is not so well defined, possibly due to surface roughness and/or chemical interaction of the silver with the atmosphere.

The predicted SPP mode positions are based on planar interface calculations. For modulated surfaces, the dispersion of SPP modes will be altered, a stop band may open up around $k_{\|}=0[8,19]$. Further analysis of our data (not shown) reveals a modest stop band, of order $\sim 2 \%$ for the silver/glass SPP. We are not aware of any quantitative calculations for the SPP dispersion appropriate for our structure, but this would be interesting to explore. We also note enhanced transmission $\omega / c 2 \pi \approx$ $1.6 \mu \mathrm{m}^{-1}, k_{\|} / 2 \pi \approx 0.6 \mu \mathrm{m}^{-1}$, where the modes associated with the two surfaces are degenerate, in accord with process (iii).

To probe the system further, we measured the dispersion of the reflectance for $p$-polarized light incident from both the air and the glass sides. These data (Fig. 3) exhibit markedly different behavior from the transmittance data, and from each other. The data for light incident from the glass side are dominated by features associated with the silver/glass SPP mode, while it is only from the air side that features associated with the silver/air SPP mode are seen.

Deeper insight can be gained by combining the transmittance, $T$, and reflectance, $R$ to infer the absorbance, $A$, via $A=1-R-T$. This can only give a good measure of absorbance provided no propagating diffracted orders are allowed, i.e., the zero-order regime [below the $(-1,0)$ glass light line, Fig. 1(b)]. We show in Fig. 4(a) transmittance, reflectance, and inferred absorbance spectra for an in-plane wave vector of $0.03 \mu \mathrm{m}^{-1}$ (closer to normal incidence the detector obscured the incident beam). Data are shown for $p$-polarized light incident from both glass and air sides. Further measurements (not shown) exhibited identical results for $s$-polarized light.

From Fig. 4(a), we see that the long wavelength transmittance peak $(\sim 715 \mathrm{~nm})$ is associated with a reflectivity minimum and an absorbance maximum. Though perhaps initially surprising, this absorption feature is again consistent with the involvement of SPPs [6,7,20]. The fields at the metal surface are enhanced when incident light couples to a SPP mode, thus aiding transmission. However, the SPP mode is also damped by absorption in the metal so that enhanced absorption is expected for the same conditions that give enhanced transmission. The form of

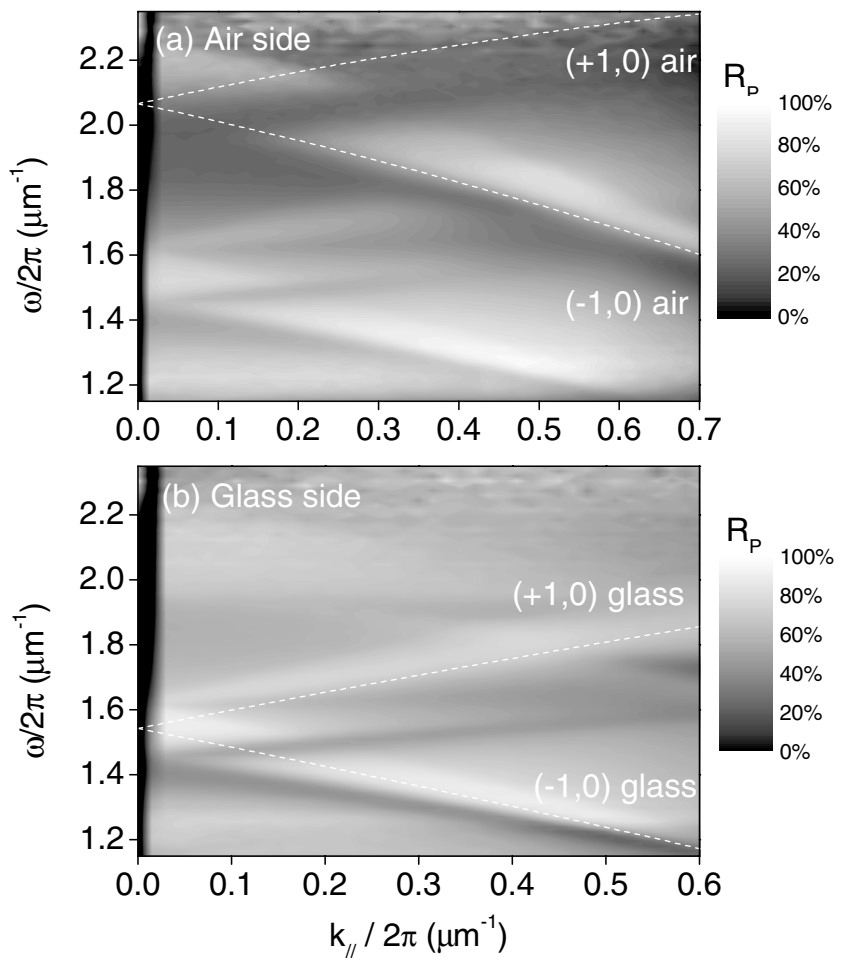

FIG. 3. Measured reflectance as a function of in-plane wave vector and frequency in the form of a grey-scale dispersion diagram for $p$-polarized incident light incident from the air side (a) and the glass side (b). Also shown are the relevant calculated SPP dispersion curves for planar surfaces. Data are shown on a linear scale. The dark vertical stripes near $k_{\|}=0$ are an experimental artifact. Arrows to indicate the offset between the experimentally derived and theoretically predicted mode dispersions have been omitted in this figure.

the reflectance, transmittance, and absorption spectra [Fig. 4(a)] and the relative position of their extrema bear a striking resemblance to recent calculations [6] (see Fig. 9 of Ref. [6]). The slight differences in positions of the different extrema are to be expected as they represent slightly different responses of the system. As a check, we also acquired data at an in-plane wave vector of $0.15 \mu \mathrm{m}^{-1}$ [Fig. 4(b)]. We see that in the zero-order region the single transmission peak of Fig. 4(a) has split into two, as expected from Fig. 2(a), and that both features correspond to reflectance minima/absorption maxima.

We also note that where the transmittance is low, around $\sim 700 \mathrm{~nm}$, the reflectance is high and the inferred absorbance is consequently low. It might be tempting to attribute the low transmittance at this wavelength to power being coupled to SPPs and, hence, lost to absorption rather than transmitted. Such a view might appear to be supported by a recent report [21] that associated the excitation of SPPs with transmittance minima. However, that work was based on slits rather than holes and, as noted above, the physics of the two situations is very different. Instead the observation of low transmittance, 


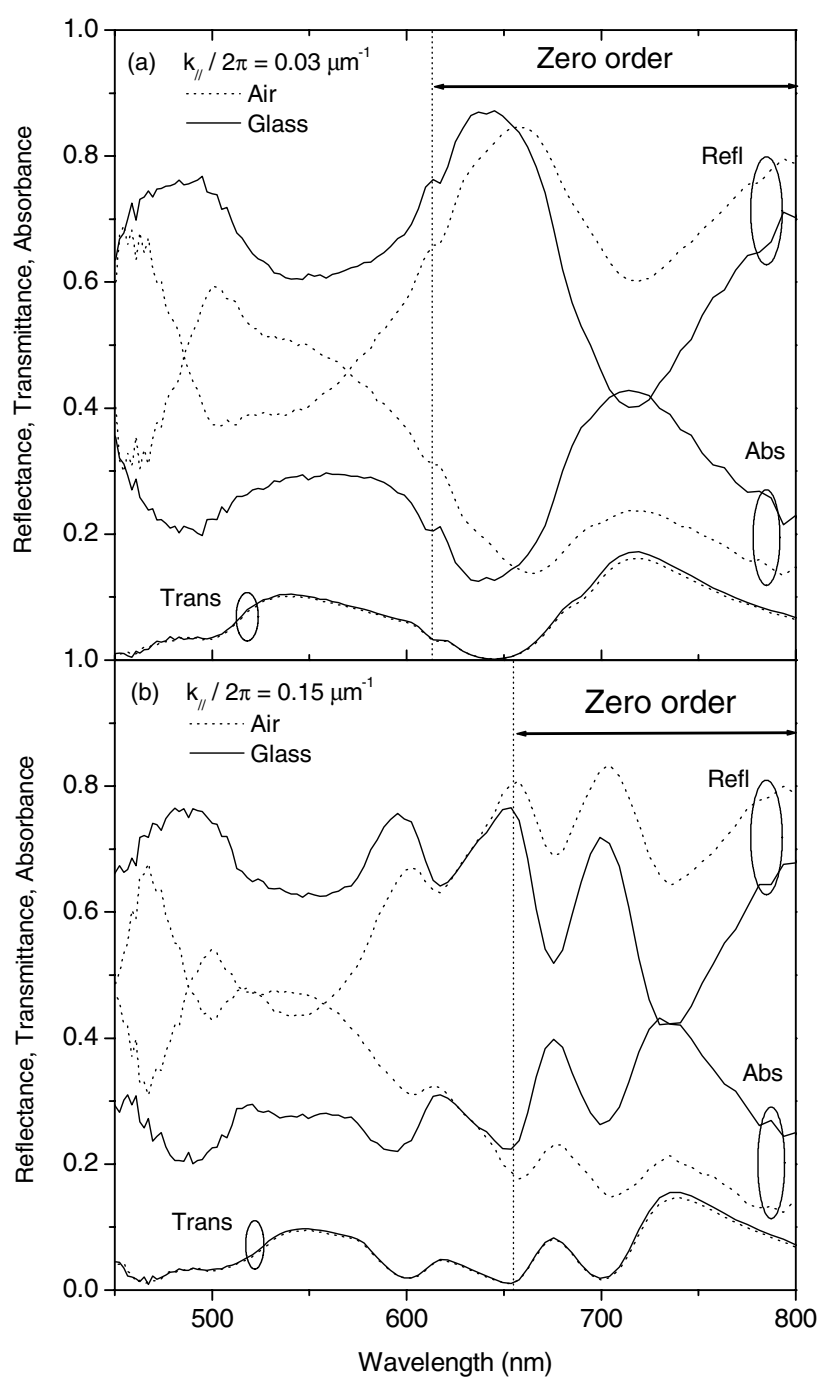

FIG. 4. Transmittance, reflectance, and inferred absorbance spectra for $p$-polarized incident light at in-plane wave vectors of $0.03 \mu \mathrm{m}^{-1}$ (a) and $0.15 \mu \mathrm{m}^{-1}$ (b).

high reflectance, and low absorbance is probably a consequence of the lack of available modes by which the transmission may be enhanced.

It is tempting to try to infer more from the absorption data, especially the feature associated with the silver/air SPP mode. However, caution must be exercised because an account needs to be taken of how much power goes into propagating diffracted orders when one is not in the zero-order regime. The importance of such diffraction can be gauged from the fact that, when conditions were such that only diffraction in the glass was allowed, we measured the fraction of incident power that goes into each of the four diffracted orders to be $\sim 5 \%$. Only in the zero-order regime can absorption data be easily interpreted.

We can summarize our findings with four observations. First, the dispersion of the transmittance features depends on the polarization of the incident light, being consistent with the involvement of $p$-polarized resonant modes [Fig. 2]. Second, the transmission and reflection spectra of metallic hole arrays are associated with modes on the two surfaces, not just for normal incidence, but for all angles [Figs. 1-3]. Third, in the zero-order regime transmission maxima are associated with reflectivity minima and with absorbance maxima [Fig. 4]. Fourth, again in the zero-order regime, the transmission minima are associated with reflectance maxima and absorbance minima [Fig. 4]. All four observations are consistent with the view that SPP modes act to enhance the fields associated with the evanescent waves, thus providing a way to increase the transmittance. In this picture, enhanced transmission naturally occurs when coupling conditions allow surface plasmon polaritons on one or both metal surfaces to be excited. We note that enhanced transmittance might perhaps also occur through the involvement of other surface waves such as surface phonon polaritons [22].

[1] T.W. Ebbesen et al., Nature (London) 391, 667 (1998).

[2] J. R. Sambles, Nature (London) 391, 641 (1998).

[3] L. Martín-Moreno et al., Phys. Rev. Lett. 86, 1114 (2001).

[4] V. M. Vigoureux, Opt. Commun. 198, 257 (2001).

[5] M. M. J. Treacy, Phys. Rev. B 66, 195105 (2002).

[6] M. Sarrazin, J. P. Vigneron, and V. M. Vigoureux, Phys. Rev. B 67, 085415 (2003).

[7] N. Bonod et al., Opt. Express 11, 482 (2003).

[8] S. A. Darmanyan and A.V. Zayats, Phys. Rev. B 67, 035424 (2003).

[9] S. Enoch et al., J. Opt. A Pure Appl. Opt. 4, S83 (2002).

[10] E. Popov et al., Phys. Rev. B 62, 16100 (2000).

[11] D. E. Grupp et al., Appl. Phys. Lett. 77, 1569 (2000).

[12] H. F. Ghaemi et al., Phys. Rev. B 58, 6779 (1998).

[13] M. G. Salt and W. L. Barnes, Opt. Commun. 166, 151 (1999).

[14] G. N. Afanasiev, J. Phys. D 34, 539 (2001).

[15] J. R. Sambles, G.W. Bradbery, and F. Yang, Contemp. Phys. 32, 173 (1991).

[16] S. C. Hohng et al., Appl. Phys. Lett. 81, 3239 (2002).

[17] W. L. Barnes, J. Mod. Opt. 45, 661 (1998).

[18] In addition to the complex refractive index of the silver [P. B. Johnson and R.W. Christy, Phys. Rev. B 6, 4370 (1972)], we included the effect of a sulfide layer at the silver/air interface typical of samples exposed to a polluted atmosphere; see G. J. Kovacs, Surf. Sci. 78, L245 (1978).

[19] W. L. Barnes et al., Phys. Rev. B 54, 6227 (1996).

[20] I. R. Hooper and J. R. Sambles, Phys. Rev. B 67, 235404 (2003).

[21] Q. Cao and P. Lalanne, Phys. Rev. Lett. 88, 057403 (2002).

[22] J. J. Greffet et al., Nature (London) 416, 61 (2002). 